\title{
MS07-P119 late | Crystal Structure Of The Crenarchaeal Membrane Family-2 Glycosyltransferase Reveals a Minimal Cellulose-Synthase Framework
}

Reichenbach, Tom (KTH Royal Institute of Technology, Stockholm, SWE)

Proteins in all three domains of life are subjected to post-translational modification via glycosylation to enable an immense number of biological functions. The majority of these constitute $N$-glycosylation of archaeal and eukaryotic proteins, where the archaeal $\mathrm{N}$-glycans is considerably more diverse compared to eukaryotes. Archaea belonging to the TACK superphylum are considered to be most similar to eukaryotes. From the few functionally characterized archaeal glycosyltransferases, it has been shown that a donor substrate, usually an NDP sugar, as well as an acceptor substrate, typically a polyisoprenyl-based lipid carrier, are needed. Here, we report the first crystal structures of a glycosyltransferase of TACK origin, namely the membrane glycosyltransferase PCManGT from $P$. calidifontis, in complex with both nucleotide and donor substrate. The structural investigation shows stabilization of the nucleotide within the active site, and further indication of interaction with the donor's mannosyl group. In the absence of a suitable acceptor substrate, our functional studies reveal enzymatic hydrolysis of the donor substrate. Furthermore, the comparison with similar 3D structures suggests that PCManGT is an enzyme that catalyzes a single glycosyl-transfer reaction to a Dol-PP-linked acceptor molecule as part of the $N$ glycan biosynthesis in this crenarcheon, rather than acting as a sugar polymerase. 